\title{
Uso do Laser ALGaInP na cicatrização de lesões cutâneas experimentais em coelhos
}

\author{
Use of ALGalnP Laser in the Wound Healing of Experimental Cutaneous Lesions in Rabbits \\ Wanessa Krüger Beheregaray', Giordano Cabral Gianotti' ${ }^{1}$, Juliano de Souza Leal' ${ }^{2}$, Fabiola de Moraes \\ Monteiro $^{3}$, Silvana Mello Simas ${ }^{3}$, Mariane Elizeire ${ }^{3}$ \& Emerson Antonio Contesini ${ }^{4}$
}

\begin{abstract}
Background: Animals with extensive cutaneous lesions remain hospitalized for long periods. In addition to increasing treatment costs, this also causes stress in these animals. Low-power laser is widely used to support tissue repair processes. Its use in the treatment of cutaneous wounds has been studied, as it reduces the wound healing period and the consequences caused by the lesion. The study aimed to evaluat the wound healing process of experimental cutaneous wounds in rabbits with the use of laser therapy.

Materials, Methods \& Results: Ten healthy New Zealand rabbits were used in the study, which were divided in two groups $(\mathrm{n}=5)$. Skin biopsies were carried out on day 16 in Group 1 and on day 8 in Group 2. The animals were under general anesthesia for the experimental cutaneous lesion to be carried out. Two $1 \mathrm{~cm}^{2}$ wounds were made on the back of each animal. The lesions were measured by a pachymeter in the immediate postoperative period, then covered with sterile gauze and maintained with rubber bandages. The animals were treated only with Tramadon $3 \mathrm{mg} \cdot \mathrm{kg}^{-1}$ twice a day, during 3 days. Only the lesions on the right side of the animals were irradiated regularly, on each side of the wound, at a distance of $0.5 \mathrm{~cm}$ from the margin. The equipment used was a 660nm wavelength AlGaInP laser diode (Laserpulse, Ibramed), pulsed at a frequency of $20 \mathrm{~Hz}$, at a dose of $3 \mathrm{~J}$ and stimulation time of $12 \mathrm{~s}$. The left-side lesions represented the control and the treatment administered to them consisted only of a cleanse using a $\mathrm{NaCl}$ solution at $0.9 \%$ once a day. The measurement of the lesion area was made every two days with a pachymeter, and the macroscopic characteristics were noted on a control card. For the histological evaluation of the wound healing process, biopsy specimens were taken from the right and left lesions on the sixteenth day in G1 and on the eighth day in G2, by means of a $6 \mathrm{~mm}$ punch. The samples were stained with Hematoxylin-eosin for the global evaluation of the tissue section, and with Masson's trichrome for evaluation of the presence of collagen fibers in interstitial fibrosis sites. Hyperemia followed by a discreet bleeding could be observed in wounds treated with laser after its application. With regard to the wound healing area, there was no difference between treated and control animals in both groups. However, in the microscopic evaluation of G1, in relation to the histological variables, significantly higher results were found in lesions treated with laser however, in the microscopic evaluation of G1, in relation to the histological variables, significantly higher results were found in lesions treated with laser: vascular proliferation, fibroblast and collagen proliferation, as well as the presence of hair follicles. In $\mathrm{G} 2$, only the fibroblastic proliferation showed a significant difference $(p=0.08)$ in lesions treated with laser.

Discussion: There was no difference in the number of inflammatory cells in this study, perhaps because the control animals underwent laser treatment of the contralateral lesion. However, the treated animals had a higher number of deposited collagen fibers than the control ones, corroborating the data mentioned in literature. The most important result found was associated with vascular proliferation, which showed a significant difference $(p=0.046)$. The formation of new blood vessels seems to be substantially facilitated by the laser use, because a relatively higher number of elements was found in the group irradiated by it, as similarly hyperemia and bleeding were observed during treatment. The results obtained suggest that irradiation with AlGaInP diode laser, at a dose of $3 \mathrm{~J}$, acted as a biostimulative agent in the wound healing of experimental lesions. Evidence of this could be demonstrated by an accelerated cell proliferation process during the experiment. The experimental lesion, animal species selected and laser application method were adequate for the execution of this project.
\end{abstract}

Keywords: wound healing, low-power-laser, rabbit, cutaneous lesions.

Descritores: cicatrização, laser de baixa potência, coelho, lesões cutâneas. 


\section{INTRODUÇÃO}

A cicatrização de feridas é um processo complexo que envolve a interação de diversos componentes celulares e bioquímicos. Desenvolve-se espontaneamente, sem intervenções externas, mas, quando tratada através de artifícios, tende a ocorrer de forma mais rápida e com melhores resultados funcionais e estéticos. A possibilidade de acelerar a cicatrização e o fechamento de lesões cutâneas mediante recursos químico-medicamentosos ou físicos tem sido objeto de investigação de inúmeros pesquisadores [19].

A utilização do laser para o tratamento de feridas cutâneas vem sendo objeto de estudo, pois promove a redução do período de cicatrização e as sequelas ocasionadas pela lesão [12]. A designação laser, na realidade, originou-se da abreviação de Light Amplification by Stimulated Emission of Radiation, cuja teoria é creditada ao físico Albert Einstein [3]. A diferença entre os vários tipos de lasers é dada pelo comprimento de onda: quanto menor o comprimento de onda, maior sua ação e poder de penetração [15]. Os benefícios citados na literatura incluem a aceleração da síntese de colágeno, o controle dos micro-organismos, aumento da vascularização, redução da dor e da inflamação [16].

O laser de baixa potencia (LBP) vem se destacando dentre os inúmeros métodos descobertos principalmente após o surgimento de equipamentos modernos, como o Alumínio-Gálio-Índio-Fósforo (AlGaInP) [3,10,18], e com eles a necessidade de maior comprovação científica de sua aplicação. Desse modo, o objetivo deste trabalho foi avaliar a cicatrização através dos achados clínicos e histopatológicos com aplicação do laser AlGaInP.

\section{MATERIAIS E MÉTODOS}

Para o experimento, foram utilizados 10 coelhos (machos e fêmeas hígidos) da raça Nova Zelândia, de origem controlada, peso de 3,5 a $4,5 \mathrm{~kg}$ e idade variando de 120 a 160 dias. Os animais foram divididos em dois grupos diferenciados somente pelo tempo de avaliação histopatológica, sendo o G1 avaliado aos 16 dias, e o G2 aos 8 dias.

Os coelhos foram submetidos à anestesia geral, tendo sido usada como medicação pré-anestésica o Ketalar ${ }^{1} 20 \mathrm{mg} \cdot \mathrm{kg}^{-1} / \mathrm{IM}$, o Dormonid ${ }^{2}$ 0,8 mg.kg-1/ IM e o Fentanest ${ }^{3}$ 0,015 mg.kg-1/IM. A indução e manutenção anestésica com Forane ${ }^{4}$ vaporizado em $\mathrm{O}_{2} 100 \%$ ao efeito. Após antissepsia, fez-se a remoção de um segmento de pele e tecido subcutâneo de $1 \mathrm{~cm}^{2}$ do lado direito e outro do esquerdo, caudal à borda da escapula, a um centímetro da coluna vertebral (Figura 1). No pós-operatório imediato, as lesões foram medidas com paquímetro, e depois cobertas com gaze estéril mantidas com atadura elástica. No pós-operatório, durante três dias, os animais recebiam o Tramadon 3 mg. $\mathrm{kg}^{-1}$ duas vezes ao dia.

Transcorridas 24 horas da indução de cada lesão, realizava-se a mensuração do comprimento e da largura, com auxílio de paquímetro; depois, cada lesão era fotografada. Os aspectos macroscópicos, como sinais flogísticos de edema, hiperemia, presença de crosta e exsudato eram observados e anotados. Após, era realizada a limpeza das lesões, tanto da direita como da esquerda, com solução de Nacl 0,9\% ${ }^{6}$ e iniciava-se o tratamento com o laser. Somente as lesões da direita foram irradiadas de maneira pontual, em cada lado da ferida, a uma distância de $0,5 \mathrm{~cm}$ da borda. O equipamento utilizado foi o laser AlGaInP, modelo Laserpulse, Ibramed ${ }^{7}$, comprimento de onda de $660 \mathrm{~nm}$, modo pulsado na frequência de $20 \mathrm{~Hz}$, na dose de $3 \mathrm{~J}$ e tempo de estimulação de $12 \mathrm{~s}$. As lesões do lado esquerdo representavam o controle, isto é, desempenhavam apenas o papel de elemento de

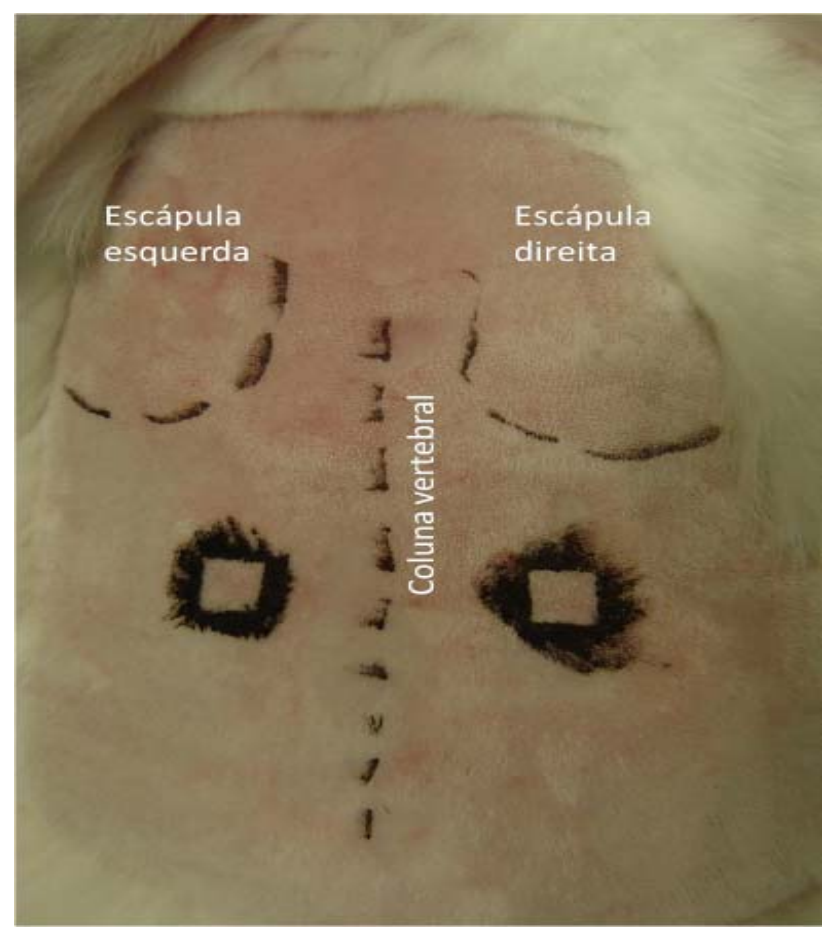

Figura 1. Delimitação do local a serem realizadas a remoção do segmento de pele de $1 \mathrm{~cm}^{2}$ do lado direito e esquerdo do dorso do coelho, a um centímetro da coluna vertebral e da borda da escápula. 
comparação e, para tanto, o único tratamento que recebiam diariamente era a limpeza com solução de Nacl $0,9 \%$. A terapia com laser era aplicada a cada dois dias, sempre precedida pela mensuração da área das lesões e registro das características macroscópicas.

Para a avaliação histológica da qualidade da cicatrização através de exame microscopia óptica, foram coletadas biópsias das feridas, através do uso de punch de $6 \mathrm{~mm}$, das lesões da direita e esquerda, no décimo sexto dia para o G1, e no oitavo para o G2.

As amostras coletadas permaneceram conservadas por 48 horas em formol tamponado $10 \%$. A seguir, foram inclusas em parafina, sendo, então, submetidas, com micrótomo, a cortes transversais de $4 \mu$. Foram coradas com hematoxilina-eosina (HE), para avaliação global dos cortes de tecido, e com Tricômico de Masson (TM), para avaliar a presença de fibras colágenas existentes nos locais de fibrose do interstício. As variáveis histológicas avaliadas, conforme descrito por Garros et al. [5], foram: proliferação vascular, células mononucleares e polimorfo-nucleares, proliferação fibroblástica, colagenização, reepitelização. Além dessas, a presença de folículos pilosos e queratina, que se

Tabela 1. A intensidade dos achados histológicos para a folículos pilosos e queratina em coloração de hematoxilinaeosina (HE) e Tricômico de Masson (TM) das biópsias cutâneas de coelhos aos 16 dias (G1) e aos 8 dias(G2) foram analisadas segundo os critérios listados abaixo.

\begin{tabular}{lllll}
\hline & \multicolumn{1}{c}{ Ausente } & \multicolumn{1}{c}{ Discreta } & \multicolumn{1}{c}{ Moderada } & \multicolumn{1}{c}{ Intensa } \\
\hline $\begin{array}{l}\text { Folículos } \\
\text { Pilosos }\end{array}$ & $\begin{array}{l}\text { Sem evidência de } \\
\text { Folículos Pilosos }\end{array}$ & $\begin{array}{l}\text { Esparsos } \\
\text { Folículos Pilosos }\end{array}$ & $\begin{array}{l}\text { Moderada quantidade } \\
\text { de Folículos Pilosos }\end{array}$ & $\begin{array}{l}\text { Grande quantidade } \\
\text { de Folículos Pilosos }\end{array}$ \\
Queratina & $\begin{array}{l}\text { Não havia } \\
\text { queratina sobre a a } \\
\text { epiderme }\end{array}$ & $\begin{array}{l}\text { Fina camada de } \\
\text { queratina }\end{array}$ & $\begin{array}{l}\text { Moderada quantidade } \\
\text { de queratina } \\
\text { depositada sobre a } \\
\text { epiderme }\end{array}$ & $\begin{array}{l}\text { Grande quantidade } \\
\text { de depósito }\end{array}$ \\
\hline
\end{tabular}

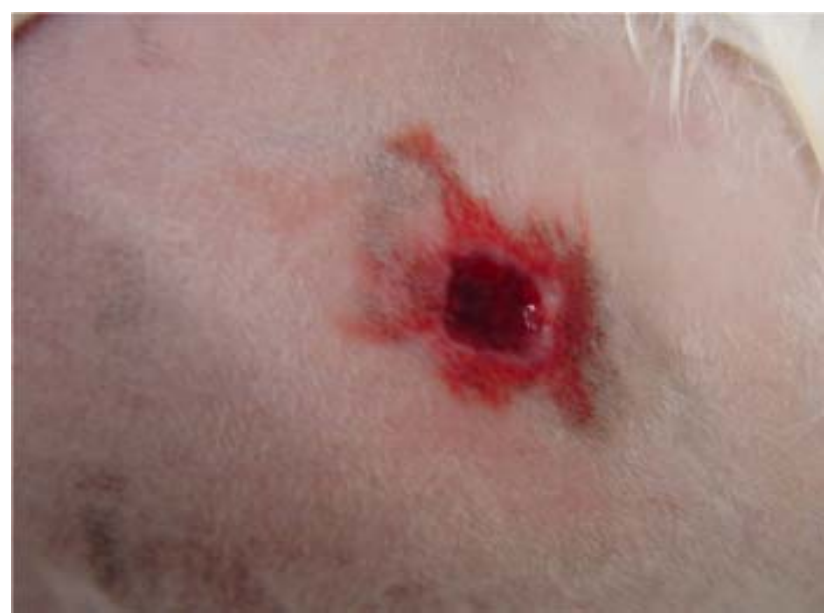

Figura 2. Pequeno sangramento observado na lesão tratada logo após a estimulação com o laser AlGaInP. encontra descrita na Tabela 1. Foram atribuídos índices histológicos para os achados: ausente, igual a zero; discreto, igual a um; moderado, igual a dois; intenso, igual a três.

A área de contração da ferida (C) foi calculada subtraindo-se a área inicial (A1), determinada no dia da indução da lesão, da área da lesão (A) nos dias 2, 4, 6, 8, 10, 12, 14 e 16, para o G1, e nos dias 2, 4, 6, 8 para o G2. Assim, C = A1 - A. A taxa de cicatrização também foi avaliada a partir desses resultados.

Avaliação estatística dos dados foi feita pelo programa Statistical Package for the Social Sciences (SPSS). As variáveis histológicas foram analisadas mediante testes não paramétricos de Mann-Whitney e de Wilcoxon. O primeiro foi usado para analisar as diferenças de médias entre amostras independentes (animais diferentes) comparando-se o lado direito e o esquerdo do $\mathrm{G} 1$, respectivamente, com o direito e o esquerdo do G2. O segundo comparou as médias de duas amostras (direita e esquerda) do mesmo animal no G1 e no G2. Ainda, com o teste de Wilcoxon foram comparadas as médias da área de cicatrização diária do lado direito e do lado esquerdo de cada grupo. $\mathrm{O}$ nível de significância utilizado para rejeitar a hipótese de nulidade foi de $10 \%(p<0,1)$.

\section{RESULTADOS}

O procedimento cirúrgico de todos os animais transcorreu sem complicações. Não houve óbitos. A recuperação anestésica e a analgesia pós-operatória mostraram-se satisfatórias. As avaliações clínicas dos animais evidenciaram adequadas recuperação, manutenção do estado geral, presença de atividade física e disposição alimentar.

Foi observado hiperemia e, por vezes, até sangramento das lesões durante o tratamento (Figura 2). Também houve crostas ressecadas cobrindo todas as lesões, porém as tratadas apresentavam essas crostas escurecidas (Figura 3).

As lesões experimentais, de maneira geral, não apresentavam exsudação, não tendo ocorrido nenhum caso de contaminação bacteriana.

Inicialmente, a área da ferida, em alguns animais, aumentou e após diminuiu gradativamente com o passar dos dias, tendo-se constatado o fechamento completo das lesões até o $15^{\circ}$ dia.

As médias das taxas de cicatrização da lesão tratada e da lesão de controle, obtidas através da mensuração realizada a cada dois dias no G1 e G2, 


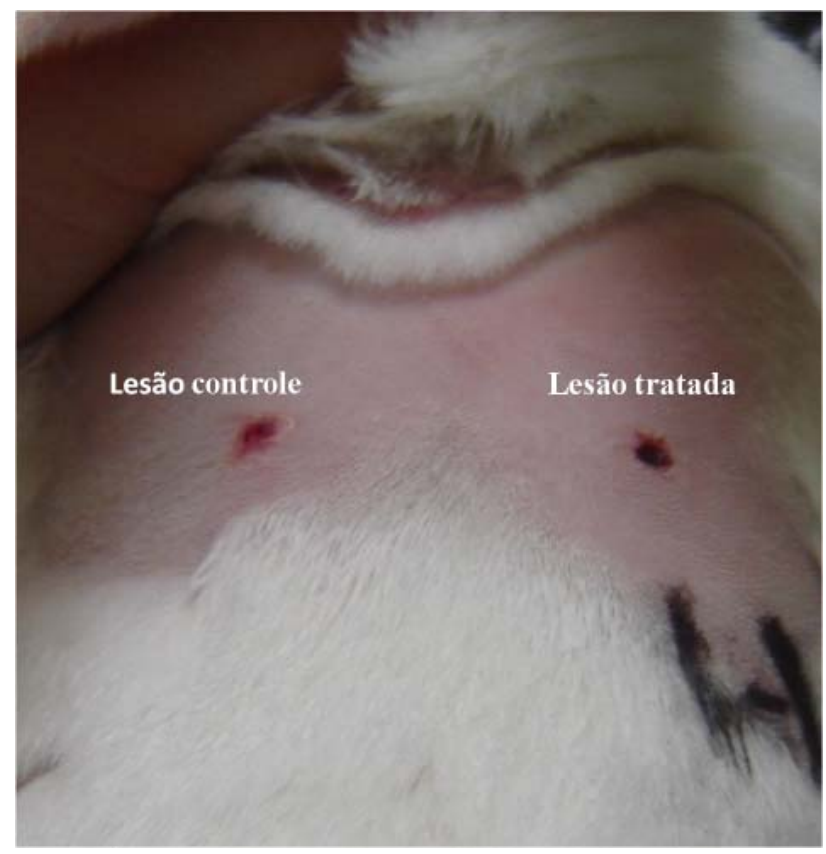

Figura 3. Lesão tratada apresentando crosta escurecida em comparação com a controle que se apresentava avermelhada.

estão demonstradas na Figura 4. Como pode ser notada, a taxa de cicatrização aumentou progressivamente, porém não foram observadas diferenças significativas entre as lesões de controle e as tratadas.

$\mathrm{Na}$ avaliação microscópica, os resultados do G1 estão dispostos na Tabela 2. Foi encontrado resultado significativamente superior nos tratados para proliferação vascular, proliferação fibroblástica, colagenização e presença de folículos pilosos. Já no G2, foi observada diferença significativa somente na proliferação fibroblástica $(p=0,08)$ das lesões tratadas. Ainda no G2, as variáveis histológicas dos folículos pilosos e queratina, tanto dos animais tratados como dos de controle, foram igual a zero.

Tabela 2. Achados histopatológico do G1 (16 dias) comparando as médias da lesão tratada com laser e da controle e o valor de significância (p)

\begin{tabular}{lccc}
\hline Variáveis Histológicos & Tratada & Controle & Valor p \\
\hline Proliferação Vascular & 2,14 & 1,78 & $0,04^{*}$ \\
Células mononucleares & 1,57 & 1,56 & 0,78 \\
$\begin{array}{l}\text { Células } \\
\text { Polimorfonucleares }\end{array}$ & 1,43 & 1,89 & 0,15 \\
Proliferação Fibroblástica & 2,29 & 2,11 & $0,10^{*}$ \\
Colagenização & 1,29 & 1,22 & $0,10^{*}$ \\
Reepitelização & 1,57 & 1,22 & 0,56 \\
Folículos Pilosos & 0,43 & 0,33 & $0,10^{*}$ \\
Queratina & 0,86 & 0,78 & 0,56
\end{tabular}

* Existe diferença significativa a $10 \%$

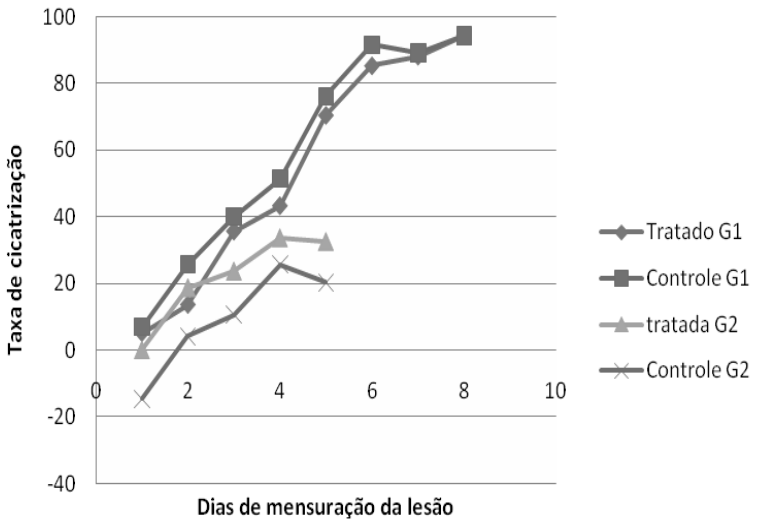

Figura 4. Gráfico mostrando a média da área de cicatrização de cada mensuração do G1 e do G2. Não houve diferença significativa entre o tempo de cicatrização da lesão tratada com laser e da controle.

\section{DISCUSSÃO}

Optou-se pela contenção manual dos animais durante as estimulações, porque o procedimento não produziria dor ou incômodo [3]. No presente trabalho foram tomadas todas as precauções para evitar o estresse, contando-se ainda com o temperamento dócil dos coelhos, o que facilitou a manipulação diária sem necessidade de anestesia.

O dorso do animal foi o local escolhido para indução da ferida para evitar a irritação por contato ou automutilação [3,9]. As escápulas e coluna vertebral foram utilizadas como referenciais anatômicos visando à padronização da região de indução das lesões em todos os animais. Nessas condições, evitou-se uma variação na irrigação sanguínea local, que, caso ocorresse, poderia determinar diferenças no processo cicatricial.

Foram realizadas duas lesões cirúrgicas em cada animal, para tentar minimizar as diferenças individuais na cicatrização e assim melhor comparar a evolução da cicatrização da lesão tratada com a da lesão de controle. Porém, Bisht et al. [2] desenvolveram semelhante metodologia em ratos e relataram que mesmo que a maioria dos efeitos do laser sejam predominantemente locais, os efeitos bioestimu-latórios podem ser observados na ferida contralateral não irradiada. Suspeita-se que fatores teciduais liberados da ferida irradiada na circulação sanguínea podem ter um efeito no local da ferida contralateral. Esse efeito sistêmico pode provocar uma falha na observação dos efeitos significantes ao comparar o tecido tratado com laser com o do lado oposto do mesmo 
animal $[3,8]$. Ainda, foi afirmado por certos autores que o LBP apresenta efeito sistêmico, pois, através de radiações não locais, outras doenças apresentam melhora [3,17].

O laser visível de $632.8 \mathrm{~nm}$ de comprimento parece ser o mais efetivo para a cicatrização de feridas $[7,16]$. Atualmente, novos instrumentos estão disponíveis no mercado, possibilitando otimizar as respostas dos tecidos superficiais. Esses equipamentos, além de oferecerem melhores respostas do que o HeNe pela proximidade do comprimento de onda, permitem irradiações durante intervalos de tempo menores porque apresentam maior potência média. Dentre esses lançamentos, encontra-se o emissor AlGaInP e o AsGa, com potências, respectivamente, $30 \mathrm{~mW}$ e 15 a $30 \mathrm{~mW}$, contra uma potência de $2 \mathrm{~mW}$ do HeNe [3]. Conforme já referido, maior potência reduz o tempo de estimulação. Devido às suas características e ao fato de poucas pesquisas terem sido realizadas até o momento com o AlGaInP, este equipamento foi o escolhido para a realização deste experimento.

A energia do laser é absorvida apenas por uma fina camada de tecido adjacente além do ponto atingido pela radiação. Esta é a razão pela qual é recomendada a aplicação de lasers de baixo poder de penetração, além de a aplicação ser pontual à lesão [3,4,15,21]. Desse modo, optou-se, neste trabalho, pela aplicação pontual nas bordas da lesão, como forma de estimulação. Contudo, há ainda trabalhos que utilizam o método de varredura acompanhado da aplicação pontual [10] ou não para estimulação [23].

Apesar do grande número de publicações sobre as ações do LBP, os seus mecanismos de ação ainda não estão bem esclarecidos, assim como a dose a ser utilizada, o intervalo de tempo de aplicação do laser, a densidade de energia, a frequência das aplicações, as características do aparelho e a profundidade do tecido tratado $[1,3,21]$. Em um trabalho utilizando LBP em ratos foi comparada a frequência da aplicação do tratamento: diária, do $1^{\circ}$ ao $6^{\circ}$ dia; a cada dois dias; somente no $1^{\circ}$ dia; somente no $3^{\circ}$ dia; somente no $5^{\circ}$ dia. Concluíram que os melhores resultados foram obtidos no tratamento a cada dois dias [23]. Por essa razão, foi estabelecido para este estudo que a frequência de estimulação aconteceria a cada dois dias.

A literatura científica sobre o uso do laser AlGaInP é reduzida. Schindl et al. [18], em 2003, tornaram-se os pioneiros no estudo dos efeitos bioestimulatórios dessa aplicação de laser através da análise dose-dependente, utilizando dose de 2, 4, 8 $\mathrm{J} / \mathrm{cm}^{2}$, na veia umbilical humana. Observou-se que houve efeito dose-dependente, pois a proliferação de células endoteliais foi diretamente proporcional ao aumento da dose utilizada [3]. Porém, em outro estudo, foram analisados o efeito do laser AlGaInP na dose de 4 e $8 \mathrm{~J} / \mathrm{cm}^{2}$ na cicatrização de feridas induzidas em ratos. Verificaram que o tratamento com $4 \mathrm{~J} / \mathrm{cm}^{2}$ foi mais eficaz que o de $8 \mathrm{~J} / \mathrm{cm}^{2}$, pois apresentou maior proliferação vascular e manteve uma maior redução do diâmetro da área da ferida em todos os instantes da análise. Ainda, observaram nos tratados que a extensão do edema e o número de células inflamatórias reduziram-se precocemente, além de ter sido induzido o aumento de fibras de colágeno depositadas, quando comparadas com o controle [11]. Neste estudo, não foi observado diferença no número de células inflamatórias, talvez porque a lesão controle tivesse sofrido influência do tratamento do laser da contralateral. Porém, o número de fibras de colágeno depositadas, assim como no estudo anteriormente citado, foi superior na tratada em comparação com o controle.

Em um estudo usando LBP (660 nm) em feridas experimentais em ratos, na dose de $3 \mathrm{~J} / \mathrm{cm}^{2}$, durante 7 dias consecutivos, constatou-se a eficácia na reepitelização dos tecidos, tanto em animais jovens como nos de mais idade [4]. Resultado semelhante foi encontrado em um experimento com coelhos, em que a dose de $3 \mathrm{~J} / \mathrm{cm}^{2}$ acelerou a reepi-telização relativamente ao controle [7]. Esses estudos serviram como referência para a escolha da dose de $3 \mathrm{~J}$ na presente pesquisa. Levou-se também em consideração o fato de outros autores terem avaliado doses de 2 e 4J $[11,18,20]$ : optou-se por uma dose intermediária, para avaliação de seus benefícios.

Em relação à área de cicatrização, não foi encontrada diferença significativa entre os tratados e controles do mesmo grupo. Esse resultado também foi encontrado em um trabalho com ratos que foram submetidos ao laser AsGaAl, com doses de 0,5, 1,5 e $4 \mathrm{~J} / \mathrm{cm}^{2}$, em que não houve diferença significativa na redução do diâmetro da ferida entre os grupos [22].

$\mathrm{O}$ resultado mais significativo encontrado foi em relação à proliferação vascular, com diferença significativa $(p=0,046)$. A formação de novos vasos parece ser substancialmente favorecida pela aplicação do laser, pois um número relativamente maior de elementos de 
pequeno e médio calibre é observado no grupo irradiado. Hiperemia e sangramentos também puderam ser observados durante o tratamento. Tatarunas et al. [20] constataram que no $15^{\circ}$ dia de irradiação com o laser AsGa, houve completa revascularização de feridas cirúrgicas, o que poderia influenciar positivamente em tecidos com déficit vascular. Avaliando a ação do LBP no pega do enxerto cutâneo autólogo em cães, um grupo de estudo observou a presença de coloração rosada mais precoce nos enxertos irradiados. Atribuíram isso ao efeito bioestimulador do laser que provoca estímulo microcirculatório, além de, possivelmente, permitir que as reanastomoses vasculares ocorressem a partir de 24 horas após a cirurgia [14].

Em nível celular, a exposição à radiação causa efeitos nos eritrócitos, leucócitos, fibroblastos e queratinócitos. $\mathrm{O}$ aumento da proliferação dos tecidos conectivos ocorre através da energia absorvida pelos aminoácidos e ácidos do núcleo, ou por bioestimulação dos cromóforos, incluindo a hemoglobina, melanina e dos citocromos mitocondriais [21]. É ao mecanismo que atribuímos a observação do escurecimento da crosta que recobria as lesões tratadas.

Em relação à reepitelização, não houve diferença estatística significativa, porém foi observada média superior das tratadas em relação aos controles, tanto no G1 como no G2. Talvez essa diferença pudesse ter sido observada se os grupos fossem maiores. Ou ainda, se as lesões tratadas e as controles fossem em animais diferentes, pois pode ter havido uma influência sistêmica, como já citado anteriormente [8,17]. A regeneração epitelial é citada por diversos autores como um dos atributos da terapia a laser [6]. Em um estudo avaliando os achados histopatológicos das lesões experimentais em ratos tratadas com laser AlGaInP, os autores observaram aceleração no processo de regeneração da epiderme e reparação da derme [6].

\section{CONCLUSÕES}

Com base nos dados observados neste trabalho é possível sugerir que a irradiação pontual do laser AlGaInP, na dose de $3 \mathrm{~J} / \mathrm{cm}^{2}$ atuou como agente bioestimulador na cicatrização de lesões cirúrgicas experimentais. Isso pode ser comprovado pela implementação acelerada do processo proliferativo celular, resultando em reparo completo durante o período de tratamento testado.

Agradecimentos. Ao Hospital de Clínicas Veterinárias da UFRGS, por ceder o aparelho de laser e permitir o desenvolvimento do projeto em suas instalações.

\section{NOTAS INFORMATIVAS}

${ }^{1}$ Ketalar- Pfizer Brasil, Guarulhos, SP.

${ }^{2}$ Dormonid- ROCHE Brasil, Jacarepaguá, RJ.

${ }^{3}$ Fentanest- Cristália Rodovia Itapira, km 14, Itapira, SP.

${ }^{4}$ Isoforine - Cristália Rodovia Itapira, km 14, Itapira, SP.

${ }^{5}$ Tramadon- Cristália, Rodovia Itapira, km 14, Itapira, SP.

${ }^{6}$ Solução de Cloreto de Sódio 0,9\% - Indústria FarmacêuticaTexon Ltda, Viamão, RS.

${ }^{7}$ Laserpulse- Indústria Brasileira de Equipamentos Médicos LTDA, Amparo, SP

\section{REFERÊNCIAS}

1 Al-Watban F.A, Zhang X.Y., Andres B.L. \& Al-Anize A. 2008. Visible Lasers were better than invisible lasers in accelerating burn healing on diabetic rats. Photomedicine and Laser Surgery. 26 (Suppl 5): 1-4.

2 Bisht D., Mehrotra, R. Singh P.A. \& Kumar A. 1999. Effect of helium-neon laser on wound healing. Indian Journal of Experimental Biology. 37 (Suppl 2): 187-189.

3 Botot B.A. 2005. Análise do Laser AlGaInP no processo de reparação tecidual de lesões cutâneas. 86f. Piracicaba, SP. Dissertação (Mestrado em Fisioterapia) - Programa de Pós-Graduação em Fisioterapia, Universidade Metodista de Piracicaba.

4 Ferreira M.A. 2006. Efeito do laser de baixa intensidade no processo de cicatrização em ratos jovens e idosos: estudo morfométrico e morfológico. 61f. Alfenas, MG. Dissertação (Mestrado em Ciência da Saúde) - Programa de Pós-Graduação em Ciências da Saúde, Universidade José do Rosário Vellano.

5 Garros I.C., Campos A.C., Tâmbara E.M., Tenório S.B., Torres O.J.M., Agulham A.M., Araújo A.C.F., Santis-Isolan P.M.B., Oliveira R.M. \& Arruda E.C.M. 2006. Extrato de Passiflora edulis na cicatrização de feridas cutâneas abertas em ratos: estudo morfológico e histológico. Acta Cirúrgica Brasileira. 21 (Supl 3): 55-65.

66 Gál P., Vidinsky B., Toporcer T., Mokry M., Mozes T., Longauer F. \& Sabo J. 2006. Histological assessment of the effect of laser irradiation on skin wound healing in rats. Photomedicine and Laser Surgery. 24 (Suppl 4): 480-488.

7 Gul N.Y., Topal A., Cangul T. \& Yanik K. 2007. Effect of TCC and laser on wound healing. Veterinary Dermatology. 19 (Suppl 1): 7-14. 
8 Jayasree R., Gupta A., Rathinam K., Mohanan P. \& Mohanty M. 2001. The influence of photodynamic therapy in wound healing process in rats. Journal of Biomaterial Applications. 15 (Suppl 3): 176-186.

9 Kashyap A., Beezhold D. \& Wiseman J. 1995. Effect of povidine iodine dermatologic ointment on wound healing. American surgeon. 61 (Suppl 6): 486-491.

10 Marcon K. \& André E.S. 2005. Efeitos do laser AlGaInP no processo de cicatrização de feridas induzidas em ratos. Revista de Fisioterapia da FURB. 1 (Suppl 1): 1-8.

11 Medrado A.R., Pugliese L.S. \& Reis S.R. 2003. Influence of low-level laser therapy on wound healing and its biological action upon myofibroblasts. Lasers in Surgery and Medicine. 32 (Suppl 3): 239-244.

12 Nakasone A., Shibata S., Suzuki S., Yamashita Y., Ohyama K. 2007. Wound healing in naso-labial region. Oral Diseases. 13 (Suppl 1): 45-50.

13 Nascimento D.G., Fernandes C.A.M., Sartoretto J.L., Brusch L.C., Cuman R.K.N. \& Silva F.P. 2006. Efeitos da radiação com o Laser HeNe 632.8 nm sobre a cicatrização de feridas em ratos. Ciência, Cuidado e Saúde. 5 (Suppl 2): $229-235$.

14 Paim C.B.V., Raiser A.G., Cardoso E. \& Beck C. 2002. Enxerto autólogo de pele, em malha, com espessura completa, na reparação de feridas carpometacarpianas de cães. Resposta à irradiação laser AsGa. Ciência Rural. 32 (Suppl 3): $451-457$.

15 Posten W., Wrone D.A, Dover J.S., Kenneth F., Arndt A., Silapunt S. \& Alam M. 2005. Low-level laser therapy for wound healing: Mechanism and efficacy. Dermatology Surgery. 31 (Suppl 3): 334-340.

16 Rocha J.S. 2004. Terapia laser, cicatrização tecidual e angiogenese. Revista Brasileira em Promoção da Saúde. 17 (Suppl 1): 44-48.

17 Rochkind S., Rousso M., Nissan M., Vilarreal M., Barr-Nea L. \& Rees D.G. 1989. Systemic effects of low power laser irradiation on the peripheral and central nervous system, cutaneous wounds and burn. Lasers in Surgery and Medicine. 9 (Suppl 2): 174-182.

18 Schindl A., Merwald H., Schindl A., Kaun C. \& Wojt A. 2003. Direct stimulatory effect of low-intensity $670 \mathrm{~nm}$ laser irradiation on human endothelial cell proliferation. British Journal of Dermatology. 148 (Suppl 2): 334-336.

19 Swain S.F. \& Henderson R.A. 1997. Small Animal Wound Management. 2nd edn. Baltimore: Williams \& Wilkins, 444 p.

20 Tatarunas A.C., Matera J.M. \& Dagli M.L.Z. 1998. Estudo clínico e anátomopatológico da cicatrização cutânea no gato doméstico: utilização do laser de baixa potência AsGa (904 nm). Acta Cirurgica Brasileira.13 (Suppl 2): 86-93.

21 Vieira S.A.L. 2006. Efeito do laser de baixa potência na cicatrização de feridas cutâneas experimentais. 49f. Franca, SP. Dissertação (Mestrado em Promoção da Saúde) - Programa de Pós-Graduação em Promoção da Saúde, Universidade de Franca.

22 Walker M.D., Rumpf S., Baxter D., Hirst D.G. \& Lowe A.S. 2000. Influence of low-level laser therapy (660 nm) on radiation-impaired wound healing model in Murine skin. Lasers in Surgery and Medicine. 26 (Suppl 1): 41-47.

23 Yasukawa A., Ohrui H., Koyama Y., Nagai M. \& Takakuda. 2007. The effect of LLLT with HeNe Laser on operative wound healing in a rat model. The Journal of Veterinary Medical Science. 69 (Suppl 8): 799-806. 